\title{
A Novel Angle Position Detector For Application to Pattern Recognition
}

\author{
Kang-Cheng Lin and Si-Chen Lee \\ Department of Electrical Engineering \\ National Taiwan University \\ Taipei, Taiwan, Republic of China \\ Tel:-886-2-3635251, Fax:-886-2-3638247
}

\begin{abstract}
Simulating the function of biological retina using electronic hardware is the future trend for intelligent electronics. It is believed that the first three features that a biological visual system extracts from an object image are edge position, edge orientation and angle position. The contrast edge position of an object and its orientation with respect to the horizontal line can be measured from the active hollow FOur OUadrant Qrientation Detector ${ }^{1}$ (FOQUOD). Based on this device, a double FOQUOD device which can extract the angle position is proposed for the first time and has been fabricated successfully. The a-Si:H angle position detector is composed of two hollow FOQUODs packed in a concentric fashion and each FOQUOD consists of four a-Si:H p-i-n solar cells. The geometry of the top ITO electrode of this detector is shown in Fig. 1, the outer $\left(\mathrm{E}_{1 \mathrm{x}}, \mathrm{I}_{1 \mathrm{x}}\right.$, $\left.E_{1 y}, I_{1 y}\right)$ and inner $\left(E_{2 x}, I_{2 x}, E_{2 y}, I_{2 y}\right)$ hollow FOQUODs are called F1 and F2, respectively. Each pair of pin diodes, i.e., $\mathrm{E}_{1 y}\left(\mathrm{E}_{1 x}\right)$ and $\mathrm{I}_{1 \mathrm{y}}\left(\mathrm{I}_{1 \mathrm{x}}\right), \mathrm{E}_{2 \mathrm{y}}\left(\mathrm{E}_{2 x}\right)$ and $\mathrm{I}_{2 \mathrm{y}}\left(\mathrm{I}_{2 x}\right)$, are connected back-to-back, by tacking the ratio of the output photocurrent difference of these pairs of two photodiodes, i.e., $\left(E_{1 y}-I_{1 y}\right) /$ $\left(E_{1 x}-I_{1 x}\right),\left(E_{2 y}-I_{2 y}\right) /\left(E_{2 x}-I_{2 x}\right)$, the position and the orientation of a contrast edge can be extracted. When a contrast edge lies across the detector as shown in Fig. 2(a), the output signals (the short circuit currents) of $F 1$ and $F 2$ can be measured. Thus, two orientations $\theta_{1}$ and $\theta_{2}$ of the contrast edge with respect to the horizontal line are measured by $F 1$ and $F 2$, respectively, i. e., $\tan \theta_{1}=\left(E_{1 y}\right.$ $\left.I_{1 y}\right) /\left(E_{1 x}-I_{1 x}\right), \tan \theta_{2}=\left(E_{2 y}-I_{2 y}\right) /\left(E_{2 x}-I_{2 x}\right)$. Conceptually, $\theta_{1}$ is equal to $\theta_{2}$ and it is considered as a contrast edge. But if an image with an angle passing through this device as shown in Fig. 2(b), the measured orientations $\theta_{1}$ and $\theta_{2}$ are not equal and the image is considered as an angle. Therefore, the angle positions of an arbitrary image can then be extracted. Fig. 3 shows the measurement results of the error signal $\theta_{2}-\theta_{1}$ when a contrast edges with different angle with respect to the horizontal line through this device. There are five test samples whose edge orientations with respect to the horizontal line are $0^{\circ}, 18^{\circ}, 45^{\circ}, 63^{\circ}$ and $90^{\circ}$, respectively. The maximum difference value is 6 degree as shown in Fig. 3 when the contrast edge with a $63^{\circ}$ orientation passing the boundary of this device. Therefore, a $6^{\circ}$ error tolerance is given to set the boundary between the criterion of the "edge" and "angle". Fig. 4 shows the accuracies of detecting an angle feature passing through this device. During the test, the double FOQUOD is equally divided into sixteen squares, the tip of the test angle is moved sequentially to each of the center of the sixteen squares and measure the output current of the F1 and F2 cells. The angle is a combination of two contrast edges with angles $\alpha$ and $\beta$ respect to the horizontal line. There are four test samples as shown in Fig. 4 , a $90^{\circ}$ angle with $\alpha=$ $45^{\circ}$ and $\beta=-45^{\circ}$ contrast edges $\left(45^{\circ} /-45^{\circ}\right)$, a $114^{\circ}$ angle $\left(63^{\circ} /-51^{\circ}\right)$, a $46^{\circ}$ angle $\left(28^{\circ} \%-18^{\circ}\right)$ and a $90^{\circ \prime}$ angle $\left(11^{\%}-79^{\circ}\right)$. If the threshold degree for distinguishing "edge" from "angle" is set to $6^{\circ}$, the average accuracy by summing up 16 measurements are beyond $55 \%$.
\end{abstract}

\section{Reference}

[1] K. C. Lin, W. J. Sah and S. C. Lee, "The Hydrogenated Amorphous Silicon Active Hollow Four Quadrant Orientation Detector For Application to Neural Network Image Sensors," IEEE Tran. on Electron Device, vol. 41, no. 5, p. 666, 1994. 


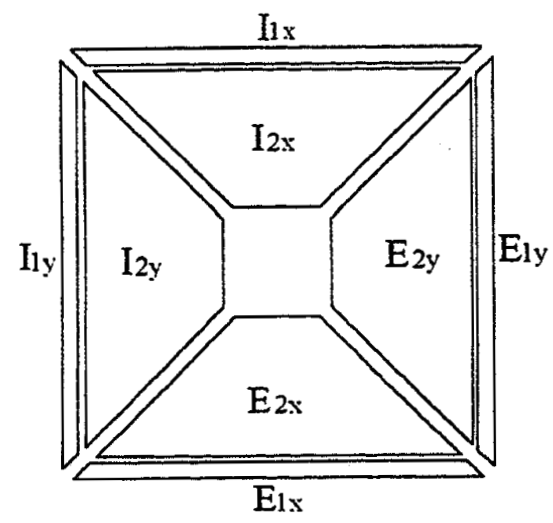

Fig. 1 The top electrode geometry of an angle position detector. It contains two hollow FOQUOD packed in a concentric fashion, the outer and inner hollow FOQUODs are called $F 1$ and $F 2$, respectively.

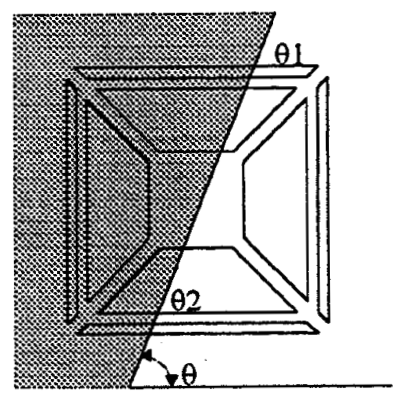

(a)

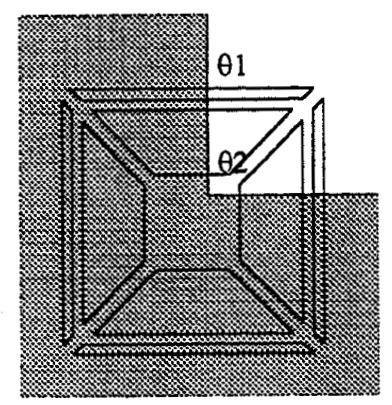

(b)

Fig. 2 (a) A contrast edge or (b) an angle lies across the detector. Two orientations $\theta_{1}$ and $\theta_{2}$ of the contrast edge with respect to the horizontal line are measured by $F 1$ and $F 2$, respectively. If $\theta_{1}$ is approximately equal to $\theta_{2}$ within $6^{\circ}$,the image is considered to be a contrast edge; otherwise the image is considered to be an angle.
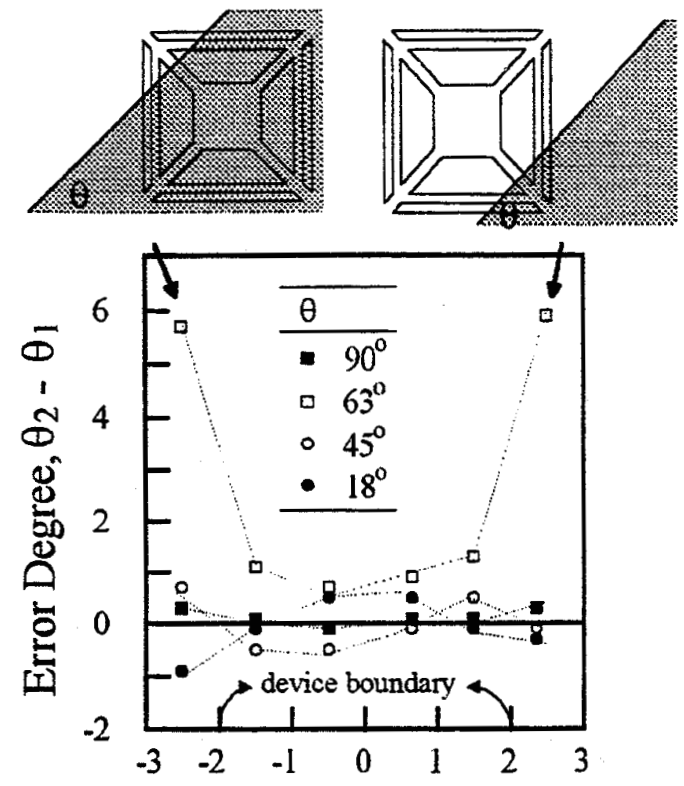

\section{Relative Position}

Fig. 3 The measurement results of error signal $\theta_{2}-\theta_{1}$ when a contrast edge with different angle $\theta$ with respect to the horizontal line, i. e., $0^{\circ}, 18^{\circ}, 45^{\circ}, 63^{\prime \prime}$ and $90^{\circ}$, respectively, moves across the device. The abscissa represents the intersection position of the edge and the horizontal line passing through the center of the FOQUOD.

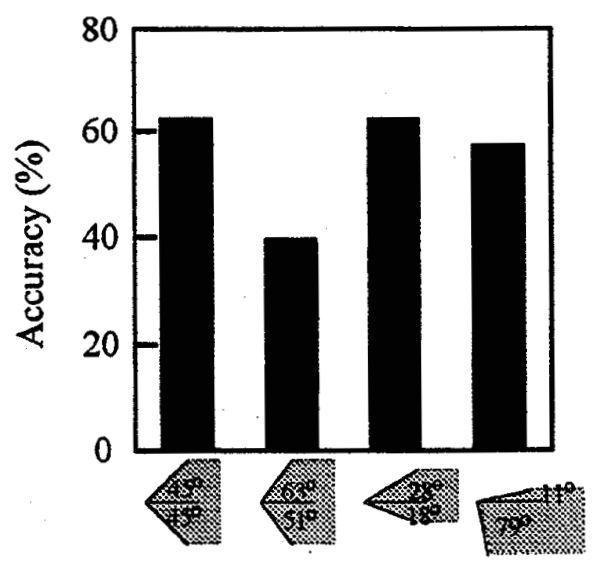

Angle Shape

Fig. 4 The accuracies of detecting an angle feature passing through the double FOQUOD. During the test, the double FOQUOD is equally divided into sixteen squares, the tip of the test angle is moved to each of the center of the sixteen squares. The 16 measured results are averaged to obtain the percentage of accuracy. 